OESOPHAGEAL CANCER

\title{
Histological precursors of oesophageal squamous cell carcinoma: results from a 13 year prospective follow up study in a high risk population
}

\author{
G-Q Wang, C C Abnet, Q Shen, K J Lewin, X-D Sun, M J Roth, Y-L Qiao, S D Mark, Z-W Dong, \\ P R Taylor, S M Dawsey
}

Gut 2005;54:187-192. doi: 10.1136/gut.2004.046631

See end of article for authors' affiliations

Correspondence to: Dr S M Dawsey, Cancer Prevention Studies Branch, Center for Cancer

Research, National Cancer Institute, 6116 Executive Boulevard, Suite 705 , Bethesda, MD 208928314, USA; dawseys@ mail.nih.gov

Revised version received 28 June 2004

Accepted for publication 26 July 2004
Background: Oesophageal squamous cell carcinoma (OSCC) has a very poor prognosis, which is largely due to late diagnosis. Successful early detection strategies will require identification of clinically relevant precursor lesions that can be targets for screening and treatment.

Aims: To identify the clinically relevant histological precursors of OSCC.

Subjects: A cohort of 682 endoscoped patients from a high risk rural population in Linxian, China.

Methods: Subjects were endoscoped and biopsied at baseline and followed for 13.5 years. We estimated the relative risk of developing OSCC for each of the initial histological diagnoses using Cox proportional hazards regression models.

Results: A total of 114 (16.7\%) patients developed OSCC during the follow up period. After adjusting for potential confounding factors, relative risks (95\% confidence intervals) for incidence of this tumour, by initial histological diagnosis, were: normal 1.0 (reference), oesophagitis 0.8 (0.2-3.2), basal cell hyperplasia 1.9 (0.8-4.5), mild dysplasia 2.9 (1.6-5.2), moderate dysplasia 9.8 (5.3-18.3), severe dysplasia 28.3 (15.3-52.3), and carcinoma in situ 34.4 (16.6-71.4).

Conclusions: In this study, squamous dysplasia and carcinoma in situ were the only histological lesions associated with a significantly increased risk of developing OSCC within 13.5 years after endoscopy. There was no evidence that oesophagitis predisposed to this tumour. Increasing grades of dysplasia were strongly associated with increasing risk, indicating that the histological grading was clinically meaningful. The follow up experience of severe dysplasia and carcinoma in situ was equivalent, suggesting that this distinction is not clinically relevant. Documenting these precursor lesions of OSCC should assist in the development of effective prevention, early detection, and treatment strategies for this disease.
$\mathrm{O}$ esophageal cancer is a common malignancy with a very poor prognosis. It is the sixth most common fatal cancer in the world, causing over 300000 deaths each year. ${ }^{1}$ Between 1992 and 1999, the five year relative survival rate for oesophageal cancer in the USA was $14.0 \%$, among the lowest for all cancers. ${ }^{2}$ The main reason for this poor prognosis is that most cases are asymptomatic and go undetected until they have spread beyond the oesophagus and are unresectable. In this setting, successful strategies for primary prevention and early detection of curable lesions are critically needed to control this disease.

Worldwide, the great majority of oesophageal cancers are oesophageal squamous cell carcinomas (OSCC). ${ }^{1}$ One important step in designing effective prevention and early detection strategies for OSCC is the identification of valid histological precursors that can be used as intermediate end points in prevention research and as targets for early detection screening and treatment. Identification and validation of such precursors requires classification of lesions by well defined histological categories and subsequent prospective follow up of biopsied patients to document which categories predict the development of OSCC.

Linxian, China, has one of the highest rates of OSCC in the world ${ }^{3}$ and therefore provides a good opportunity to evaluate possible precursors of this disease. During 1985-1991, the Cancer Institute of the Chinese Academy of Medical Sciences (CICAMS) and the US National Cancer Institute (NCI) collaborated in carrying out two nutrition intervention trials among 40-69 year old adults in Linxian. ${ }^{4-6}$ Since the end of the interventions, participants of these trials have been followed as cohorts. As part of the initial trials, an endoscopic survey was carried out in 1987 among dysplasia trial participants. ${ }^{58}$ Here we report a 13.5 year follow up study of the 682 subjects in that survey who had one or more satisfactory squamous oesophageal biopsies and were free of invasive cancer at the beginning of the follow up period. The purpose of this analysis was to examine the association between the initial squamous biopsy findings and subsequent risk of developing OSCC. The first 3.5 years of this follow up have been reported previously. ${ }^{9}$

\section{MATERIALS AND METHODS}

\section{Endoscopic survey}

Endoscopic examinations were conducted among subjects enrolled in the Linxian Dysplasia Trial, a six year randomised prospective nutrition intervention trial limited to individuals with a previous Chinese cytological diagnosis of oesophageal dysplasia. ${ }^{56}$ Active intervention, consisting of daily tablets containing 26 vitamins and minerals at 2-3 times US Recommended Daily Allowances or matched placebos, began on 1 May 1985 and ended on 30 April 1991. ${ }^{56}$

Abbreviations: $\mathrm{BCH}$, basal cell hyperplasia; CICAMS, Cancer Institute of the Chinese Academy of Medical Sciences; CIS, squamous carcinoma in situ; dysplasia NOS, dysplasia not otherwise specified; OSCC, oesophageal squamous cell carcinoma; IEN, intraepithelial neoplasia; $\mathrm{NCl}$, US National Cancer Institute 

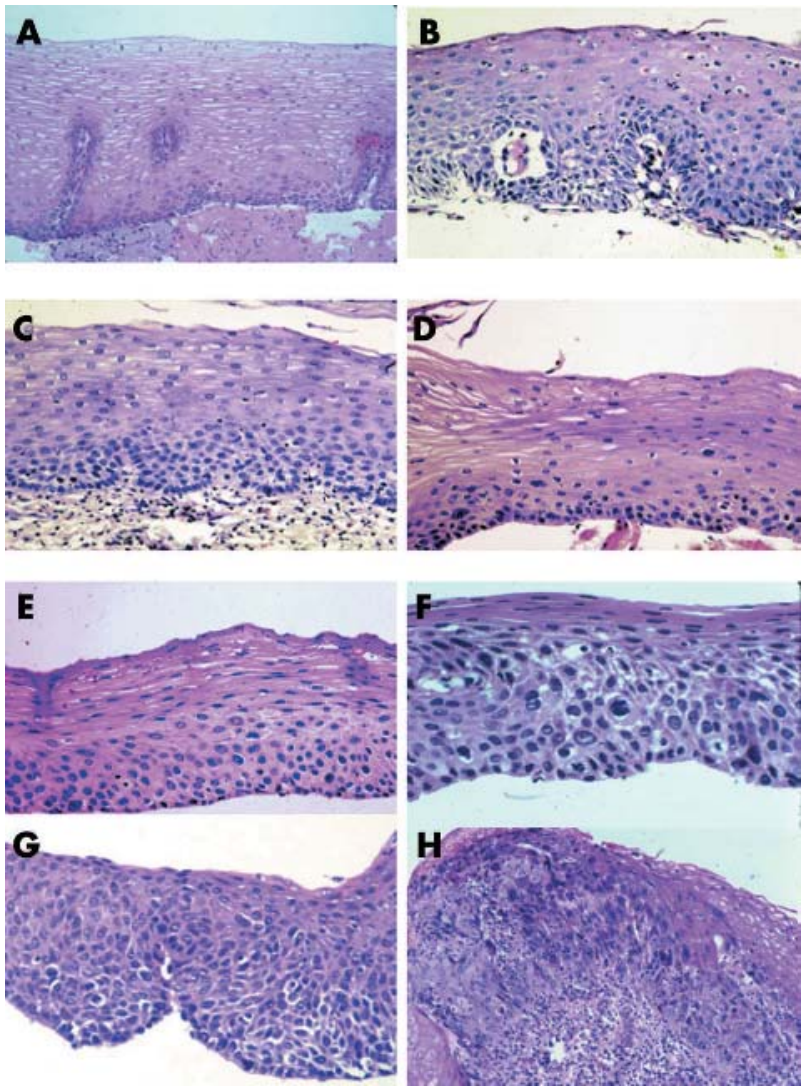

Figure 1 Histological categories. (A) Normal: basal zone thickness $<15 \%$ of epithelial thickness; papillae limited to the lower two thirds of the epithelium; no atypia. (B) Oesophagitis: epithelium infiltrated by neutrophils and eosinophils. (C) Basal cell hyperplasia: basal zone thickness $>15 \%$ of epithelial thickness; no atypia. (D) Mild dysplasia: atypical cells in the lower third of the epithelium. (E) Moderate dysplasia: atypical cells in the lower two thirds of the epithelium. (F) Severe dysplasia: atypical cells in all thirds of the epithelium but not full thickness. (G) Carcinoma in situ: full thickness involvement by atypical cells, without invasion. (H) Squamous cell carcinoma: early invasion into the lamina propria.

In November and December 1987, after 30 months of active intervention, 833 Dysplasia Trial subjects underwent endoscopy. The study was approved by the human research review committee of the CICAMS and the institutional review board of the NCI, and informed consent was obtained from each subject prior to the procedure. Details of subject selection, refusal rates, and participant characteristics have been previously described. ${ }^{7}$

During endoscopy, the entire oesophagus and stomach were visually examined, and one or more $2.8 \mathrm{~mm}$ biopsies were taken from all focal lesions. If no focal lesions were found, a standard site in the mid-oesophagus was sampled. No standard sampling of the stomach was done. Biopsies were fixed in $10 \%$ buffered formalin or $95 \%$ ethanol, embedded in paraffin, cut into $5 \mu \mathrm{m}$ sections, and stained with haematoxylin and eosin.

\section{Histological categories}

The biopsy slides were read jointly by three pathologists (QS, KJL, SMD), without knowledge of the patient's history, treatment group, or the visual endoscopic findings. Histological criteria were based on previous descriptions. ${ }^{8}$ Examples of the major histological categories are shown in fig 1.

\section{Normal}

A stratified squamous epithelium was present which showed no features diagnostic of the other histological categories listed below. Mature squamous cells with abundant clear cytoplasm, scattered lymphocytes, and compressed nuclear fragments ("squiggle cells") were occasionally seen in the epithelium. The lamina propria, if present, commonly contained a few scattered mononuclear inflammatory cells.

\section{Acanthosis}

An otherwise normal epithelium was $\geqslant 0.5 \mathrm{~mm}$ thick.

\section{Oesophagitis}

One or more of the following three criteria were present: elongation of lamina propria papillae into the upper third of the epithelium together with basal cell hyperplasia $(\mathrm{BCH})$ $>15 \%$ of total epithelial thickness; epithelial infiltration by neutrophils or eosinophils; or a dense non-follicular infiltrate of mononuclear inflammatory cells or neutrophils in the lamina propria.

\section{Basal cell hyperplasia $(\mathrm{BCH})$}

An otherwise normal epithelium had a basal zone thickness $>15 \%$ of total epithelial thickness, without elongation of lamina propria papillae or other abnormality.

\section{Squamous dysplasia}

Nuclear atypia (enlargement, pleomorphism, and hyperchromasia), loss of normal cell polarity, and abnormal tissue maturation were present in the lower third (mild), in the lower two thirds (moderate), or in all thirds (severe) of the epithelium, without full thickness involvement or invasion. Dysplastic biopsies which could not be graded because of biopsy size or orientation were categorised as squamous dysplasia, not otherwise specified (NOS).

\section{Squamous carcinoma in situ (CIS)}

Dysplastic squamous cells were present throughout the full thickness of the epithelium, without invasion.

\section{Squamous cell carcinoma}

Neoplastic squamous cells were present which had invaded through the basement membrane.

\section{Follow up procedures}

Incident cancers and deaths were identified through several methods which assured essentially complete ascertainment of these events. ${ }^{5}$ During the intervention period, all trial participants were visited each month by their village doctors to deliver the intervention pills, and all those with cancer symptoms and those who died from any cause were identified and reported. In addition, all medical facilities, including commune hospitals, the Linxian County Cancer Hospital, and the Cancer Hospital in the prefecture capital of Anyang, notified investigators of all cancer diagnoses among residents of the communes in the Dysplasia Trial. After the active intervention, information was obtained every month from village doctors on the vital status, occurrence of any incident cancer, and cause of death for each trial participant. In addition, all living participants were individually surveyed by questionnaire at the end of intervention in 1991, in 1996, and again in 2000. Throughout the trial and the post trial follow up period, symptomatic individuals were referred to the CICAMS field station in Yaocun Commune or to their commune hospital for further clinical evaluation. Case records and diagnostic materials (histology slides, cytology slides, and/or $x$ ray films) of all subjects developing cancer were reviewed and the diagnosis of cancer confirmed by members of an International Endpoints Review Committee 
Table 1 Incidence and relative risk of oesophageal squamous cell carcinoma during 1987-2001, by initial histological diagnosis

\begin{tabular}{lccccc}
\hline $\begin{array}{l}1987 \\
\text { diagnosis }\end{array}$ & $\begin{array}{l}\text { No of } \\
\text { subjects }\end{array}$ & $\begin{array}{l}\text { No of } \\
\text { OSCC cases }\end{array}$ & $\begin{array}{l}\text { Cumulative OSCC } \\
\text { incidence (\%) }\end{array}$ & $\begin{array}{l}\text { OSCC } \\
\text { incidence rate* }\end{array}$ & $\begin{array}{l}\text { Relative risk (95\% Cl) } \\
\text { of OSCC incidence† }\end{array}$ \\
\hline Normal & 375 & 31 & 8.3 & 605 & 1.0 (ref) \\
Acanthosis & 77 & 6 & 7.8 & 597 & $0.9(0.4-2.2)$ \\
BCH & 40 & 6 & 15.0 & 1637 & $1.9(0.8-4.5)$ \\
Oesophagitis & 33 & 2 & 6.1 & 493 & $0.8(0.2-3.2)$ \\
mD & 76 & 18 & 23.7 & 1518 & $2.9(1.6-5.2)$ \\
MD & 30 & 15 & 50.0 & 10507 & $9.8(5.3-18.3)$ \\
SD & 23 & 17 & 73.9 & 21364 & $28.3(15.3-52.3)$ \\
NOS & 12 & 7 & 58.3 & 7689 & $12.7(5.5-29.6)$ \\
CIS & 16 & 12 & 75.0 & 21194 & $34.4(16.6-71.4)$ \\
Total & 682 & 114 & 16.7 & 1510 & na \\
\hline
\end{tabular}

$\mathrm{BCH}$, basal cell hyperplasia; mD, mild dysplasia; $M D$, moderate dysplasia; SD, severe dysplasia; NOS, dysplasia not otherwise specified; CIS, carcinoma in situ; OSCC, oesophageal squamous cell carcinoma.

*Oesophageal cancer incidence rates (per 100000 person years) adjusted to the age distribution of the analytic cohort at baseline $(<50$ years $=22.4 \% ; 50-59$ years $=46.0 \% ; \geqslant 60$ years $=31.5 \%)$.

†Relative risk (95\% confidence interval (CI)) for OSCC adjusted for age, sex, tobacco use, alcohol use, 1983 cytology diagnosis, and treatment group.

composed of expert pathologists, cytologists, and radiologists from both the US and CICAMS. ${ }^{5}$

\section{Analysis}

In the 1987 endoscopic survey, 754 of 833 endoscoped subjects had one or more squamous biopsies which were satisfactory for histological diagnosis. Of these, 72 had received a diagnosis of cancer (of any site) before or during the 1987 endoscopy examinations. The analytic cohort for this study consisted of the remaining 682 subjects who had at least one satisfactory squamous biopsy and were free of invasive cancer at the beginning of the follow up period.

The age, sex, smoking status (ever smoked regularly $\geqslant 6$ months), and alcohol use (any drinking of alcohol during the last 12 months) were recorded by questionnaire. The pretrial cytology diagnoses (dysplasia 1 (low grade); dysplasia 2 (high grade)) came from balloon cytology examinations performed in $1983 .{ }^{10}$ For each subject, a worst oesophageal diagnosis was determined using the hierarchy of invasive cancer $>$ carcinoma in situ $>$ dysplasia (graded) $>$ oesophagitis $>$ acanthosis $>$ BCH $>$ normal.

Descriptive statistics of the follow up results were generated based on all subjects in the analytic cohort, with deletions as necessary for missing data. Age adjusted

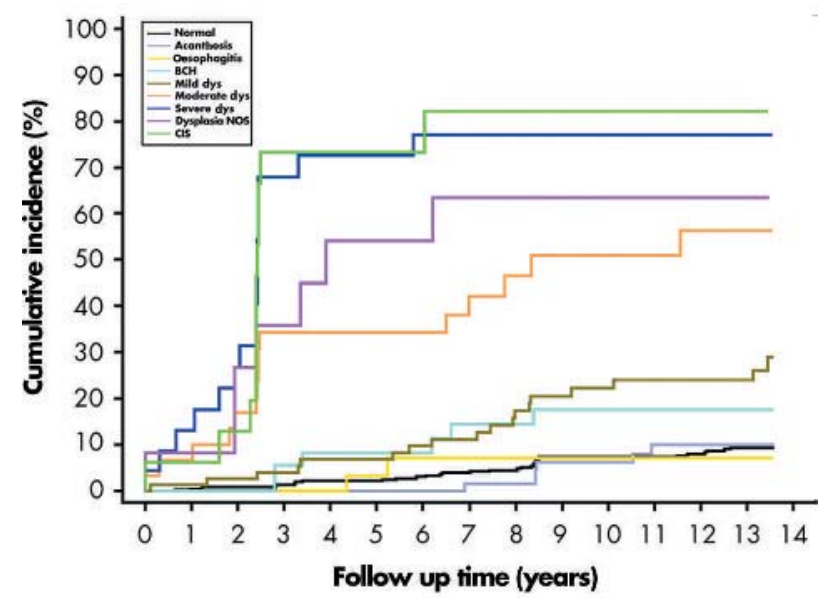

Figure 2 Cumulative incidence of oesophageal squamous cell carcinoma by initial histological diagnosis. BCH, basal cell hyperplasia; Mild dys, mild dysplasia; Moderate dys, moderate dysplasia; Severe dys, severe dysplasia; Dysplasia NOS, dysplasia not otherwise specified; CIS, carcinoma in situ. oesophageal cancer incidence rates for each of the histological categories were calculated by determining the number of cases which had occurred in each age and histological category and dividing that number by the number of person years of observation in that category. Rates were age adjusted using the 1987 age distribution of all subjects in the analytic cohort as weights $(<50$ years $=22.4 \% ; 50-59$ years $=46.0 \%$; $\geqslant 60$ years $=31.5 \%$ ). A cumulative incidence plot was generated using the Kaplan-Meier method by plotting ( $1-$ the survival function) versus follow up time. Relative risks and 95\% confidence intervals (CI) for the histological categories (modelled as indicator variables with normal as the reference) were estimated by Cox proportional hazards regression models using SAS PHREG (SAS Institute, Cary, North Carolina, USA) with adjustment for age (continuous variable), sex, smoking, alcohol use, 1983 cytology diagnosis, and treatment group (dichotomous variables).

\section{RESULTS}

Baseline characteristics of the analytic cohort have been described previously. ${ }^{9}$ There was little variation in age across the diagnostic categories but subjects with dysplasia or carcinoma in situ were more likely to be male, to smoke, to have a 1983 cytology diagnosis of dysplasia 2, and to be in the placebo treatment group than were subjects with a normal mucosa.

There were 114 new cases of OSCC diagnosed in the analytic cohort during the follow up period, including 52 cases during the intervention period (1987-1991), 48 cases during the next five years (1991-1996), and 14 cases during the last five years (1996-2001). There was a total cumulative incidence of $16.7 \%$ during the follow up period, and a crude incidence rate of 1510 cases per 100000 person years of follow up.

Table 1 and fig 2 show the relationship between the 1987 oesophageal biopsy diagnoses and OSCC incidence during the 13.5 year follow up period. Eight per cent of patients with a normal mucosa or acanthosis and $6 \%$ of those with a worst biopsy diagnosis of oesophagitis developed OSCC in the follow up period. Fifteen per cent of patients with $\mathrm{BCH}$ developed OSCC. In contrast, $24 \%$ of those with mild dysplasia, $50 \%$ of those with moderate dysplasia, and $74 \%$ of those with severe dysplasia developed OSCC during this time. Seventy five per cent of patients whose initial biopsies showed CIS developed OSCC, the same proportion as those who started the follow up period with severe dysplasia. The cumulative incidence for patients originally classified as dysplasia NOS was $58 \%$, consistent with this category being a 
mix of all grades of dysplasia. The age adjusted incidence rates and multivariate adjusted relative risks for developing OSCC paralleled the cumulative incidence values. Relative to those with a normal mucosa, only subjects with initial biopsies showing squamous dysplasia or carcinoma in situ had a significantly elevated risk. Exclusion of cases diagnosed in the first year after endoscopy did not significantly change the relative risk estimates $(\mathrm{BCH}=2.0$, mild dysplasia $=2.9$, moderate dysplasia $=9.6$, severe dysplasia $=25.7$, dysplasia NOS $=11.5$, and carcinoma in situ $=34.5$ ).

In addition to the 114 OSCC, there were 60 gastric adenocarcinomas (including 53 from the gastric cardia) and 13 other cancers (six liver, four lung, one breast, one colon, and one rectum) diagnosed in the analytic cohort during the follow up period. There were 266 deaths in the analytic cohort during this time, including 85 from OSCC, 37 from gastric cardia cancer, 15 from other cancers, 36 from heart disease, 60 from stroke, and 33 from other causes. Of the 30 patients with an initial diagnosis of moderate dysplasia, 15 developed OSCC, 10 died of other causes, and five were alive without evidence of cancer at the end of the follow up period. Of the 39 patients with initial severe dysplasia or CIS, 29 developed OSCC, four died of other causes, one was alive with gastric cardia cancer, and five were alive without evidence of cancer at the end of the follow up period.

\section{DISCUSSION}

Identification of clinically relevant precursor lesions is a prerequisite for designing successful prevention and early detection strategies for OSCC because such lesions are the targets for prevention and early detection efforts. Clinically relevant precursor lesions, which may be histological or molecular lesions, are abnormalities that precede invasive OSCC by months to years and are reliably associated with an increased risk of tumour development. Once documented, such lesions can be used for risk stratification of individuals, they can be gold standards for evaluating primary screening tests, and they can be targets for endoscopic therapy. They can also potentially be used as intermediate end points in prevention studies such as chemoprevention trials.

Relatively little has been written about the histological precursors of OSCC in low risk Western populations. Squamous dysplasia (including carcinoma in situ) is thought to be the relevant lesion because it is the accepted precursor in other organs with squamous epithelia, such as the cervix, and is commonly found adjacent to foci of invasive OSCC in oesophagectomy specimens. ${ }^{11} 12$

Most studies of OSCC precursor lesions have been performed in high risk Asian populations. Crespi et al and Munoz and colleagues ${ }^{13-15}$ described a high prevalence of histological oesophagitis, atrophy, and dysplasia in endoscopic surveys of Iranian and Chinese populations which have high OSCC rates and little or no evidence of oesophageal reflux. Based on differences in the prevalence of these lesions between these high risk populations and a low risk Chinese population and a one year follow up study of 20 patients, they proposed that oesophagitis, atrophy, and dysplasia may be precursor lesions of OSCC in these groups. ${ }^{14}{ }^{15}$ Using similar methods, Yang and Qiu ${ }^{16}{ }^{17}$ reported no difference in the prevalence of oesophagitis or the prevalence of atrophy in high versus low risk Chinese populations but found a higher prevalence of $\mathrm{BCH}$ and dysplasia in their high risk group. They also re-endoscoped 186 patients 30-78 months after their initial surveys and found progression to cancer in $34 \%$ of subjects whose initial biopsies showed both oesophagitis and dysplasia but similar progression to cancer in only $4 \%$ of those whose original biopsies showed oesophagitis alone, leading them to suggest that dysplasia is the primary precancerous lesion in the high risk areas of China. ${ }^{16}{ }^{17}$
Others have since referred to Yang and Qiu's comparative population findings to propose that $\mathrm{BCH}$ is also a precursor lesion of OSCC, preceding dysplasia in the carcinogenic sequence. ${ }^{18}$

The current study is the largest and longest prospective follow up of an endoscoped cohort looking for the development of OSCC. Our findings show no evidence that oesophagitis is associated with an increased risk of developing OSCC within 13.5 years after the initial diagnosis (relative risk 0.8 (95\% CI $0.2-3.2)$ ) and so we believe it is unlikely to be a significant precursor lesion in this population. Our initial endoscopic survey found no examples of atrophy $^{8}$ so we cannot comment on this lesion. It is noteworthy however that Yang and Qiu also found this lesion to be rare in their surveys, ${ }^{16}{ }^{17}$ making it unlikely to be an important precursor lesion.

We found a nearly twofold increased risk of OSCC associated with an initial diagnosis of $\mathrm{BCH}$ (relative risk 1.9 (95\% CI 0.8-4.5)). Although this increased risk was not statistically significant, the cumulative incidence curve (fig l) was very similar to that of mild dysplasia, and it is our experience that the morphological distinction between $\mathrm{BCH}$ and mild dysplasia can be difficult. Thus we think that patients with a diagnosis of $\mathrm{BCH}$ should be viewed clinically with some increased concern, even if only because of the risk of histological misclassification between this diagnosis and mild dysplasia.

In our analysis, all grades of squamous dysplasia and carcinoma in situ were associated with a significantly increased risk of developing OSCC, identifying these lesions as histological precursors of this tumour. In addition, we found that increasing grades of dysplasia were associated with dramatically increasing risk, implying real biological differences in these histological categories. Thus we believe that stratifying treatment based on dysplasia grade is appropriate, and that a shift in dysplasia grade over time (during passive follow up or during an intervention) may confer a real change in risk. The follow up experience of severe dysplasia and carcinoma in situ was equivalent in this study, suggesting that this diagnostic distinction is not clinically relevant and should be abandoned. This is not surprising, considering that common artefacts such as retention of surface epithelium in the biopsy forceps and imperfect biopsy orientation during tissue processing can significantly influence whether dysplastic cells appear to reach the upper third or the full thickness of the epithelium. ${ }^{8}$

Previous reports have questioned the utility of histological grading of oesophageal squamous intraepithelial neoplasia (IEN). ${ }^{19}$ In the current study, higher dysplasia grades identified distinctly higher cancer risks, showing that clinically useful histological grading of oesophageal squamous IEN is possible, using the extent of epithelial involvement by dysplastic cells as the basis for grading. Although occasional cases of invasive OSCC can be seen in which only the lower layers of the overlying epithelium contain dysplastic cells, the current findings demonstrate that over a series of cases, grading by extent of epithelial involvement correctly predicts different levels of OSCC risk. Accurate grading by this system requires good biopsy orientation, so the distribution of dysplastic cells from the base to the surface of the epithelium can be evaluated, but this can be reproducibly achieved by spreading the biopsies on supporting material in the endoscopy suite before fixation. ${ }^{20}$

Recent publications have advocated a unified histological classification for epithelial neoplasia throughout the gastrointestinal tract, including a two grade (low grade, high grade) classification of intraepithelial neoplasia. ${ }^{21}$ While the major findings of this study (significant OSCC risk associated with 
squamous IEN and increasing risk associated with increasing IEN grade) would probably be the same in such a two grade IEN classification, we believe that important information would also be lost. In the current study, the risk estimates for the three grades of dysplasia differed greatly, with little or no overlap of confidence intervals between adjacent grades, suggesting that three different grade-specific clinical responses may be appropriate. For example, mild dysplasia might be re-evaluated periodically or treated by chemoprevention, moderate dysplasia might be followed more closely or treated by focal endoscopic therapy, and severe dysplasia/ CIS should be treated by endoscopic or surgical therapy without delay. A three grade classification of dysplasia is also supported by the results of a recent chemoprevention trial in Linxian in which patients were diagnosed by the same histological categories used in the current study. In this trial, selenomethionine had a significant beneficial effect on patients who began the trial with mild but not moderate dysplasia, ${ }^{22}$ indicating that it may be important to reserve a separate diagnostic category for individuals with the earliest morphological evidence of squamous neoplasia who may be more responsive to medical interventions.

All patients in this study had a Chinese cytological diagnosis of dysplasia four years prior to their 1987 endoscopy examination. Previous studies have shown that Chinese cytological examinations in the 1980s had high sensitivity (approximately 90\%) but low specificity (approximately 20\%) for identifying individuals with histological squamous dysplasia, ${ }^{23}$ so it is not surprising that many of the patients who had a positive cytological diagnosis in 1983 showed no dysplasia in their endoscopic biopsies in 1987.

The 1987 endoscopic survey did not use iodine staining to highlight abnormal mucosal areas and therefore it is probable that some dysplastic lesions were missed. These missed lesions may have contributed to the cancer incidence in patients initially diagnosed as normal who later developed OSCC during the follow up period. If so, such initial misclassification would likely cause this analysis to underestimate the true relative risks associated with having dysplastic lesions. A later endoscopic survey in Linxian that used mucosal iodine staining showed that iodine unstained lesions had sensitivities of $63 \%, 93 \%, 96 \%$, and $100 \%$ for identifying foci of mild dysplasia, moderate dysplasia, severe dysplasia/CIS, and early invasive OSCC, respectively. ${ }^{24}$ This ability to visualise and target the documented precursor lesions of OSCC should allow more accurate diagnosis and risk stratification of patients, targeted focal therapy, and more accurate evaluation of medical (for example, chemoprevention) and surgical (for example, endoscopic therapy) interventions aimed at preventing the development of invasive OSCC.

This study was carried out in Linxian, China, which has one of the highest rates of OSCC in the world but we believe that the results are also applicable to lower risk populations. Exposure to tobacco and alcohol, which is responsible for most cases of OSCC in low risk areas, is not common in Linxian, but this population appears to be exposed to tobacco related carcinogens, such as polycyclic aromatic hydrocarbons, from other sources. ${ }^{25}$ As noted above, squamous dysplasia is the most likely precursor of OSCC in low risk groups and there is no evidence to suggest that dysplasia acts differently in different populations. Once early OSCC has developed, its behaviour and clinical progression appear similar in both high and low risk groups. ${ }^{26}$ Thus we believe that the significance and behaviour of squamous dysplasia are probably similar in all populations.

In summary, in this study squamous dysplasia and carcinoma in situ were the only histological lesions that predicted the development of OSCC in this high risk population within 13.5 years of follow up observation. There was no evidence that oesophagitis predisposed to OSCC during this time. Increasing grades of dysplasia were strongly associated with increasing risk, indicating that this precursor lesion can be divided morphologically into clinically meaningful grades. The follow up experience of severe dysplasia and CIS was equivalent, suggesting that this distinction is not clinically relevant. Documenting these precursor lesions of OSCC should assist in the development of effective prevention, early detection, and treatment strategies for this disease.

\section{ACKNOWLEDGEMENTS}

This research was supported by intramural NCI funds and by NIH contracts N01-SC-91030, N01-CP-40540, 263-MQ-822420, and 263MQ-731789.

\section{Authors' affiliations}

G-Q Wang, X-D Sun, Y-L Qiao, Z-W Dong, Cancer Institute, Chinese Academy of Medical Sciences, Beijing, China

C C Abnet, M J Roth, P R Taylor, S M Dawsey, Cancer Prevention Studies Branch, Center for Cancer Research, National Cancer Institute, Bethesda, Maryland, USA

S D Mark, Biostatistics Branch, Division of Cancer Epidemiology and Genetics, National Cancer Institute, Bethesda, Maryland, USA

Q Shen, Henan Medical University, Zhengzhou, China

K J Lewin, Department of Pathology, UCLA Center for the Health Sciences, Los Angeles, California, USA

Correspondence may also be addressed to Dr Y-L Qiao, Cancer Institute, Chinese Academy of Medical Sciences, 17 S Panjiayuan Lane, Chaoyang District, PO Box 2258, Beijing 100021, Peoples' Republic of China; qiaoy@public.bta.net.cn

Conflict of interest: None declared.

\section{REFERENCES}

1 Parkin DM, Bray Fl, Devesa SS. Cancer burden in the year 2000. The global picture. Eur J Cancer 2001;37(suppl 8):S4-66

2 Ries LAG, Eisner MP, Kosary CL, et al. eds. SEER cancer statistics review, 1975-2000. Bethesda, MD: National Cancer Institute, 2003.

3 Munoz N, Day NE. Esophageal cancer. In: Schottenfeld D, Fraumeni JF Jr, eds. Cancer epidemiology and prevention. New York: Oxford University Press, 1996:681-706

4 Blot WJ, Li J-Y, Taylor PR, et al. Nutrition intervention trials in Linxian, China: supplementation with specific vitamin/mineral combinations, cancer incidence, and disease-specific mortality in the general population. J Natl Cancer Inst 1993:85: 1483-92.

5 Li B, Taylor PR, Li J-Y, et al. Linxian nutrition intervention trials. Design, methods, participant characteristics, and compliance. Ann Epidemiol 1993;3:577-85.

6 Li JY, Taylor PR, Li B, et al. Nutrition intervention trials in Linxian, China: multiple vitamin/mineral supplementation, cancer incidence, and diseasespecific mortality among adults with esophageal dysplasia. J Natl Cancer Inst 1993;85: 1492-8.

7 Dawsey SM, Wang GQ, Taylor PR, et al. Effects of vitamin/mineral supplementation on the prevalence of histological dysplasia and early cancer of the esophagus and stomach: results from the Dysplasia Trial in Linxian, China. Cancer Epidemiol Biomarkers Prev 1994;3:167-72.

8 Dawsey SM, Lewin KJ, Liu FS, et al. Esophageal morphology from Linxian, China. Squamous histologic findings in 754 patients. Cancer 1994;73:2027-37.

9 Dawsey SM, Lewin KJ, Wang GQ, et al. Squamous esophageal histology and subsequent risk of squamous cell carcinoma of the esophagus. A prospective follow-up study from Linxian, China. Cancer 1994;74:1686-92.

10 Shen Q, Liu SF, Dawsey SM, et al. Cytologic screening for esophageal cancer: results from 12,877 subjects from a high-risk population in China. Int J Cancer 1993;54:185-8.

11 Mandard AM, Marnay J, Gignoux M, et al. Cancer of the esophagus and associated lesions: detailed pathologic study of 100 esophagectomy specimens. Hum Pathol 1984; 15:660-9.

12 Ohta H, Nakazawa S, Segawa K, et al. Distribution of epithelial dysplasia in the cancerous esophagus. Scand J Gastroenterol 1986;21:392-8.

13 Crespi M, Munoz N, Grassi A, et al. Oesophageal lesions in northern Iran: a premalignant condition? Lancet 1979;2:217-21.

14 Crespi M, Munoz N, Grassi A, et al. Precursor lesions of oesophageal cancer in a low-risk population in China: comparison with high-risk populations. Int J Cancer 1984;34:599-602.

15 Munoz N, Crespi M, Grassi A, et al. Precursor lesions of oesophageal cancer in high-risk populations in Iran and China. Lancet 1982;1:876-9. 
16 Guanrei Y, Songliang Q. Endoscopic surveys in high-risk and low-risk populations for esophageal cancer in China with special reference to precursors of esophageal cancer. Endoscopy 1987;19:91-5.

17 Qiu SL, Yang GR. Precursor lesions of esophageal cancer in high-risk populations in Henan Province, China. Cancer 1988:62:551-7.

18 Wang LD, Qiu SL, Yang GR, et al. A randomized double-blind intervention study on the effect of calcium supplementation on esophageal precancerous lesions in a high-risk population in China. Cancer Epidemiol Biomarkers Prev 1993:2:71-8

19 Watanabe H, Tada T, Iwafuchi M, et al. Does esophageal dysplasia really exist? Stomach Intestine $1991 ; 26: 133-40$.

20 Weinstein WM. Handling of the biopsy specimen prior to immersion in fixatives. In: Lewin KJ, Riddell RH, Weinstein WM, eds. Gastrointestinal pathology and its clinical implications. New York: Igaku-Shoin, 1992:9-11.

21 Schlemper RJ, Riddell RH, Kato Y, et al. The Vienna classification of gastrointestinal epithelial neoplasia. Gut 2000;47:251-5.
22 Limburg PJ, Qiao Y-L, Mark SD, et al. Helicobacter pylori seropositivity and subsite-specific cancer risks in Linxian, China. J Natl Cancer Inst $2001 ; 93: 226-33$

23 Dawsey SM, Shen Q, Neiberg RK, et al. Studies of esophageal balloon cytology in Linxian, China. Cancer Epidemiol Biomarkers Prev 1997;6: 121-30.

24 Dawsey SM, Fleischer DE, Wang GQ, et al. Mucosal iodine staining improves endoscopic visualization of squamous dysplasia and squamous cell carcinoma of the esophagus in Linxian, China. Cancer 1998:83:220-31.

25 Roth MD, Qiao YL, Rothman N, et al. High urine 1-hydroxypyrene glucuronide concentrations in Linxian, China, an area of high risk for squamous oesophageal cancer. Biomarkers 2001;6:381-6.

26 Lewin KJ, Dawsey SM, Wang GQ. Squamous carcinoma of the esophagus in China and the West: are they different disorders? Dis Esophagus 1995;8:44-7.

\section{EDITOR'S QUIZ: GI SNAPSHOT}

\section{Black oesophagus-cause?}

\section{Clinical presentation}

A 64 year old woman was admitted with coffee ground emesis. She had been bedridden for the previous two years due to osteoarthritis but had no past history of upper gastrointestinal ulcer, gastro-oesophageal reflux disease, or corrosive intake. Initial upper endoscopy revealed a patchy black appearance of the oesophageal mucosa extending from the proximal third of the oesophagus, with adherent yellow exudates, which became circumferential in the distal third of the oesophagus (fig 1). The black mucosa showed a sharp boundary at the gastro-oesophageal mucosal junction (fig 1), and the gastric and duodenal mucosa showed a normal appearance. The patient was treated with intravenous ranitidine for seven days and oral ranitidine thereafter. Endoscopy one week later revealed that the oesophageal mucosa was diffusely covered by thin whitish exudates with no stenosis, and biopsies revealed ulcerated mucosa with massive necrosis.
Robin Spiller, Editor

\section{Question}

What is the diagnosis?

See page 227 for answer

This case is submitted by:

A Sako, J Kitayama, T Inove, S Kaizaki, H Nagawa Department of Surgical Oncology, University of Tokyo, Tokyo, Japan H Suzuki Suzuki Hospital, Tokyo, Japan

Correspondence to: Dr A Sako, Department of Surgical Oncology, University of Tokyo, Hongo7-3-1, Bunkyo-ku, Tokyo 113-8655, Japan; sakou-dis@h.u-tokyo.ac.jp

doi: $10.1136 /$ gut.2004.044784
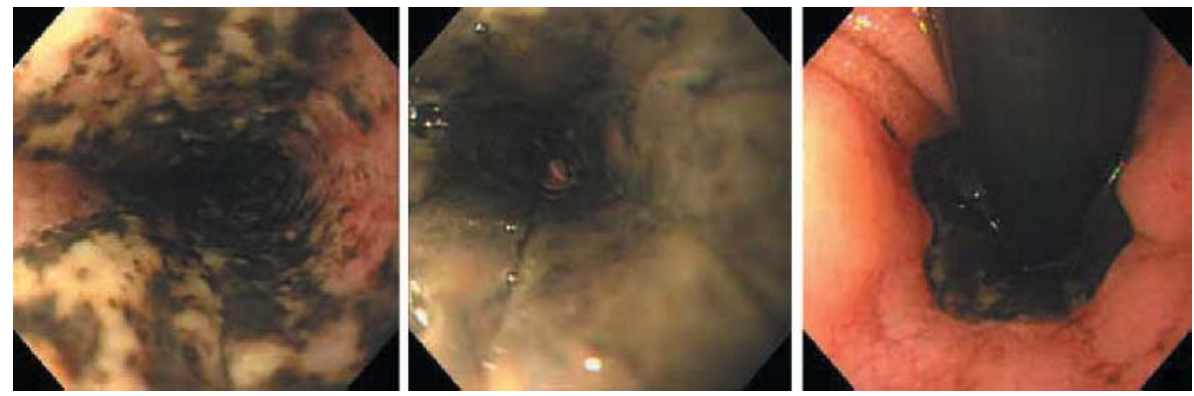

Figure 1 Upper gastrointestinal endoscopy of the oesophagus revealing a diffusely black mucosa that ends sharply at the gastro-oesophageal mucosal junction. 